\title{
Behind the Volatility Index Levels: The Paradox of 2016
}

\author{
G. D. Hancock ${ }^{1}$ \\ ${ }^{1}$ University of Missouri-St. Louis, St. Louis, USA \\ Correspondence: G. D. Hancock, University of Missouri-St. Louis, 1 University Boulevard, 559 Hickory Ridge \\ Court, St. Louis, MO 63121, USA. Tel: 314-607-5949.
}

Received: November 9, 2017

Accepted: November 30, 2017

Online Published: December 28, 2017

doi:10.20849/iref.v1i1.270

URL: https://doi.org/10.20849/iref.v1i1.270

\begin{abstract}
The low 2016 volatility index levels present a paradox in light of previous research suggesting periods of uncertainty and negative news events should reflect higher VIX levels. This study uses daily data for the VIX, VIX futures and the VVIX, to examine the information content of variations in the natural logarithmic changes in the index levels relative to 12 other parallel time periods encompassing 2004-2016. Straight-forward variation and predictive tests are constructed to determine signs of unusual market volatility behavior. The results reveal strong evidence of unusual volatility behavior during the 2016 election period, pocked by frequent periods of abnormal returns. The 2016 VIX levels alone are shown to be insufficient to draw conclusions regarding investor sentiment.
\end{abstract}

Keywords: VIX, VIX futures, VVIX, market volatility, investor sentiment, 2016 US presidential election, Volatility of Volatility, uncertainty

The low implied 30-day forward volatility levels during 2016, present a puzzle in light of previous research showing that periods of uncertainty should reflect higher Chicago Board Option's Exchange (CBOE) S\&P 500 Volatility Index (VIX) levels. The time-period investigated is striking due to the frequency of negative news reports combined with historically low VIX levels and unperturbed financial markets. During the same time period, unprecedented levels of public uncertainty and fear were regularly reported.

The 2016 election period $\mathbf{E P}_{16}$ paradox: a strong economy, strong stock market, low inflation, low unemployment and low VIX levels combined with high levels of anxiety, uncertainty and fear of the future.

The purpose of this paper is to apply a straight-forward examination of the natural logarithmic changes and variability in stock market volatility indexes to explain the seemingly byzantine behavior of the VIX during the period June 1, 2016 through November 30, 2016.

This study examines daily data over the period 2004-2016 for the VIX, VIX Futures (VX) and the Volatility of Volatility index $(\boldsymbol{V} \boldsymbol{V I X})$. The 2016 results are compared to 12 parallel time periods, representing four actual election years, 2004, 2008, 2012 and 2016, and nine parallel periods in non-election years. The findings indicate that VIX levels alone are insufficient to explain the underlying uncertainty.

\section{Background}

The impact of negative news is expected to have a negative impact on stock returns and a positive impact on VIX levels as shown by Tetlock (2007), Mamaysky and Glasserman (2017). Their findings demonstrate that increases in VIX are large following the release of negative news events. The more unusual the negative news event, the larger the increase in VIX is. Positive news events are shown to have the reverse effect on VIX levels.

In a perfect world, with no negative news, election periods are expected to have higher volatility levels due to the uncertainty of the outcome. Godell and Vahamaa (2013) study the effects of political uncertainty on implied stock market volatility during five US presidential election cycles. They document increases in VIX with changes in the probability of success of the eventual winner. The association between implied volatility and the probability of electing the eventual winner is positive even after the authors control for changes in overall election uncertainty. The findings indicate that the presidential election process engenders market anxiety as investors form and revise their expectations regarding future macroeconomic policies. VIX decreases as the winner of the US presidential election becomes more certain.

Academic research on VIX and investor sentiment largely confirms the expected relationship between 
uncertainty and the stock market. Researchers, including Whaley (2000), Traub, Ferreira, McArdle and Antogelli (2000) and Smales (2014), provide evidence in support of high VIX levels during periods of uncertainty, when investors require additional compensation in the form of above-average excess returns for riskier assets. Two studies suggest that VIX levels alone are insufficient to determine the variance premium for stock returns. The first, is a unique study by Dhaene, Dony, Forys, Linders and Shoutens (2012), that proposes a new fear index, with the moniker FIX. The quantification of the FIX takes into account: market risk (VIX), liquidity risk, systemic risk and herd behavior via the concept of comonotonicity. This approach allows the authors to measure an overall level of market uncertainty as well as to identify precisely the individual importance of the distinct risk components.

The second article, by Drechsler (2013), demonstrates that uncertainty is strongly reflected in option prices but the fluctuations in the VIX and implied volatility curve contain an important uncertainty component. The author uses a calibration of the equilibrium model to simultaneously match salient moments of consumption and dividends, the equity premium, risk-free rate, the variance premium and implied volatility skew to document the predictive power of the variance premium for stock returns. Drechsler's results imply that uncertainty and its variation are important for jointly explaining the equity premium, risk-free rate, and the large variance premium embedded in the "high" price of options. The Dhaene, et al. (2012) and the Drechsler (2013) articles indicate the need to look beyond VIX levels to determine underlying uncertainty.

\section{Data}

The VIX and VX data series begin on 3/26/2004 with the trade of the first VIX futures contract. The later introduction of the VVIX on 1/3/2007, results in 696 fewer observations for this index than VIX and VX. The last trading day for all data series is $12 / 30 / 2016$, resulting in a population of 3,214 observations for the VIX, VX indexes and 2,518 for VVIX.

The daily VX data set is constructed by rolling the nearest available contract into the next, one day prior to the delivery of the current contract. Specifically, on the first VX trading day, 3/26/2004, a position is established in the nearest available contract, the May 04. One day prior to the May 04 delivery, 5/19/04, the position is rolled into the Jun 04 contract. On 6/16/04, one day prior to the Jun 04 delivery, the contract is rolled into the Jul 04 , and so on. With the exception of the missing $\mathrm{Apr} 04$ contract, there are no further interruptions to the pattern of rolling one-month near futures throughout the 2004-2016 time-period.

Table 1 displays the average levels of $\mathrm{VIX}_{\mathrm{i}}, \mathrm{VX}_{\mathrm{i}}$ and $\mathrm{VVIX}_{\mathrm{i}}$, where $i$ is equal to each election, and parallel non-election, period $\left(\mathrm{EP}_{\mathrm{i}}\right)$. The bold values in each column represent the highest values in each election period. The 2016 VIX and VX averages are the lowest since 2006 while VVIX is at its highest level since introduction. The high volatility of VIX relative to its average value in 2016 suggests a large amount of uncertainty surrounding the 30-day forward volatility estimate of the S\&P 500 index. Column $\mathrm{E}(\mathrm{VVIX}) / \mathrm{E}\left(\mathrm{VIX}_{\mathrm{i}}\right)$ shows that the 2016 VVIX multiple of 6.7315, is the highest since the index was introduced in 2007. Similarly, column VX Premium indicates the $7.56 \%$ is also at its highest level since trading began in 2004 .

Table 1. Average Volatility

\begin{tabular}{lccccc}
\hline $\mathrm{EP}_{\mathrm{i}}$ & $\mathrm{E}(\mathrm{VIX})$ & $\mathrm{E}\left(\mathrm{VX}_{\mathrm{i}}\right)$ & $\left.\mathrm{E}(\mathrm{VVIX})_{\mathrm{i}}\right)$ & $\mathrm{E}(\mathrm{VVIX}) / \mathrm{E}\left(\mathrm{VIX}_{\mathrm{i}}\right)$ & $\mathrm{VX}_{\mathrm{i}}$ Premium \\
\hline 2004 & 15.04 & 15.80 & na & na & $5.05 \%$ \\
2005 & 12.61 & 13.38 & na & na & $6.11 \%$ \\
2006 & 13.32 & 13.74 & na & na & $3.15 \%$ \\
2007 & 20.72 & 20.65 & 91.24 & 4.4035 & $-0.34 \%$ \\
$\mathbf{2 0 0 8}$ & $\mathbf{3 6 . 8 8}$ & $\mathbf{3 3 . 8 1}$ & 88.72 & 2.4056 & $-8.32 \%$ \\
2009 & 25.64 & 26.71 & 77.58 & 3.0257 & $4.17 \%$ \\
2010 & 23.92 & 25.16 & 89.24 & 3.7308 & $5.18 \%$ \\
$\mathbf{2 0 1 1}$ & 29.21 & 28.74 & 97.64 & 3.3427 & $-1.61 \%$ \\
2012 & 17.11 & 18.40 & 92.98 & 5.4342 & $7.54 \%$ \\
2013 & 15.74 & 15.98 & 80.48 & 5.1131 & $1.52 \%$ \\
2014 & 15.87 & 16.01 & 85.18 & 5.3674 & $0.88 \%$ \\
\hline
\end{tabular}


Table 1 (continued). Average Volatility

\begin{tabular}{lccccc}
\hline $\mathrm{EP}_{\mathrm{i}}$ & $\mathrm{E}\left(\mathrm{VIX}_{\mathrm{i}}\right)$ & $\mathrm{E}\left(\mathrm{VX} \mathrm{i}_{\mathrm{i}}\right)$ & $\mathrm{E}\left(\mathrm{VVIX}_{\mathrm{i}}\right)$ & $\mathrm{E}\left(\mathrm{VVIX} \mathrm{i}_{\mathrm{i}}\right) / \mathrm{E}\left(\mathrm{VIX}_{\mathrm{i}}\right)$ & $\mathrm{VX}_{\mathrm{i}}$ Premium \\
\hline 2015 & 17.54 & 17.63 & 94.46 & 5.3854 & $0.51 \%$ \\
$\mathbf{2 0 1 6}$ & 14.56 & 15.66 & $\mathbf{9 8 . 0 1}$ & $\mathbf{6 . 7 3 1 5}$ & $\mathbf{7 . 5 6 \%}$ \\
$\mathbf{2 0 0 4 - 2 0 1 6}$ & $\mathbf{1 9 . 1 5}$ & $\mathbf{1 9 . 5 4}$ & $\mathbf{8 7 . 6 2}$ & $\mathbf{4 . 5 7 5 5}$ & $\mathbf{2 . 6 1 \%}$ \\
\hline
\end{tabular}

The data is parsed, as shown in Table 2, into four actual election periods and nine parallel non-election periods with the corresponding number of observations, $n_{i}$, shown in the last column. Each election period $\left(\mathrm{EP}_{\mathrm{i}}\right)$ is defined as the first trading day in June to the last trading day of November. The four years in bold indicate an actual US Presidential election period and the remaining nine years indicate a parallel time period in a non-election year. Both the actual and the parallel non-election periods are referred to as $\mathrm{EP}_{\mathrm{i}}$ for simplicity.

Table 2. Election Periods

\begin{tabular}{lccc}
\hline $\mathrm{EP}_{\mathrm{i}}$ & First Trade Day & Last Trade Day & $\mathrm{n}_{\mathrm{i}}$ \\
\hline $\mathbf{2 0 0 4}$ & 1-Jun & 30-Nov & $\mathbf{1 2 7}$ \\
2005 & 1-Jun & 30-Nov & 127 \\
2006 & 1-Jun & 30-Nov & 128 \\
2007 & 1-Jun & 30-Nov & 128 \\
$\mathbf{2 0 0 8}$ & 2-Jun & $\mathbf{2 8 - N o v}$ & $\mathbf{1 2 6}$ \\
2009 & 1-Jun & 30-Nov & 128 \\
2010 & 1-Jun & 30-Nov & 128 \\
2011 & 1-Jun & 30-Nov & 128 \\
$\mathbf{2 0 1 2}$ & 1-Jun & 30-Nov & $\mathbf{1 2 6}$ \\
2013 & 3-Jun & 29-Nov & 127 \\
2014 & 2-Jun & 28-Nov & 127 \\
2015 & 1-Jun & 30-Nov & 127 \\
$\mathbf{2 0 1 6}$ & 1-Jun & 30-Nov & $\mathbf{1 2 8}$ \\
\hline
\end{tabular}

Testing comparisons of $\mathrm{EP}_{16}$ are made to all other parallel time periods as well as to the populations. The populations are defined as:

1) Data Period Y $\left(\mathbf{D P}_{\mathbf{Y}}\right)$. The annual populations begin on the first trading day, through the last in each year $Y$, resulting in $n_{Y}$ observations.

2) Data Period $\mathbf{N}\left(\mathbf{D P}_{\mathbf{N}}\right)$. The global population $N$ begins on $3 / 26 / 04$ and ends on $12 / 30 / 16$, resulting in $N$ observations.

The $\mathrm{DP}_{\mathrm{Y}}$ represent a greater barrier to overcome than $\mathrm{DP}_{\mathrm{N}}$ because annual values are more closely related to disturbances caused by an election period. Table 3 below clarifies the population data periods for $\mathrm{DP}_{\mathrm{N}}$ and $\mathrm{DP}_{\mathrm{Y}}$.

Table 3. Population N and Y

\begin{tabular}{cccc}
\hline Year & $\mathrm{DP}_{\mathrm{N}}$ & $\mathrm{DP}_{\mathrm{Y}}$ & $\mathrm{n}_{\mathrm{Y}}$ \\
\hline $\mathbf{2 0 0 4}$ & $\mathbf{2 6 - M a r}$ & $\mathbf{3 / 2 6} \mathbf{- 1 2 / 3 1}$ & $\mathbf{1 9 3}^{*}$ \\
2005 & & $1 / 03-12 / 30$ & 252 \\
2006 & & $1 / 03-12 / 29$ & 251 \\
2007 & & $1 / 03-12 / 31$ & 251 \\
$\mathbf{2 0 0 8}$ & & $\mathbf{1 / 0 2}-\mathbf{1 2 / 3 1}$ & $\mathbf{2 5 3}$ \\
\hline
\end{tabular}


Table 3 (continued). Population $\mathrm{N}$ and $\mathrm{Y}$

\begin{tabular}{cccc}
\hline Year & $\mathrm{DP}_{\mathrm{N}}$ & $\mathrm{DP}_{\mathrm{Y}}$ & $\mathrm{n}_{\mathrm{Y}}$ \\
\hline 2009 & & $1 / 02-12 / 31$ & 252 \\
2010 & & $1 / 04-12 / 31$ & 252 \\
2011 & & $1 / 03-12 / 30$ & 252 \\
$\mathbf{2 0 1 2}$ & $\mathbf{N}=\mathbf{3 2 1 4}$ & $\mathbf{1 / 0 3}-\mathbf{1 2 / 3 1}$ & $\mathbf{2 5 0}$ \\
2013 & & $1 / 02-12 / 31$ & 252 \\
2014 & & $1 / 02-12 / 31$ & 252 \\
2015 & & $1 / 02-12 / 31$ & 252 \\
$\mathbf{2 0 1 6}$ & $\mathbf{3 0 - D e c}$ & $\mathbf{1 / 0 4}-\mathbf{1 2 / 3 0}$ & $\mathbf{2 5 2}$
\end{tabular}

*VX trading began on 3/26/2004. VVIX trading began on 1/3/2007.

\section{Methodology}

This section presents a common application of Variation Test (VT) and Predictive Test (PT) methodologies applied to each $X$, where $\mathrm{X}=\ln \left(\mathrm{VIX}_{\mathrm{i}, \mathrm{t}} / \mathrm{VIX}_{\mathrm{i}, \mathrm{t}-1}\right), \ln \left(\mathrm{VX}_{\mathrm{i}, \mathrm{t}} / \mathrm{VX} \mathrm{i}_{\mathrm{i}, \mathrm{t}-1}\right)$ and $\ln \left(\mathrm{VVIX}_{\mathrm{i}, \mathrm{t}} / \mathrm{VVIX} \mathrm{i}_{\mathrm{i}, \mathrm{t}-1}\right)$.

\subsection{Variation Tests (VT)}

The variation tests $(\mathrm{VT})$ are straight-forward $F$-tests comparing the sample variation $\mathrm{S}\left(\mathrm{X}_{16}\right)$, to each other sample election period $\mathrm{S}\left(\mathrm{X}_{\mathrm{i}}\right)$ and to population $d=\mathrm{DP}_{\mathrm{Y}}$ and $\mathrm{DP}_{\mathrm{N}}$.

$$
\begin{aligned}
\mathrm{VT \# 1} & =\mathrm{S}\left(\mathrm{X}_{2016}\right) / \mathrm{S}\left(\mathrm{X}_{\mathrm{i}}\right) \\
\mathrm{VT} \# 2 & =\mathrm{S}\left(\mathrm{X}_{\mathrm{i}}\right) / \sigma\left(\mathrm{X}_{\mathrm{d}}\right)
\end{aligned}
$$

$\mathrm{S}(\mathrm{Xi})$ is the sample variation of $\mathrm{Xt}$ during EPi:

$$
\left[1 /\left(n_{i}-1\right) \sum_{t_{i}=\text { Jun } 1}^{n_{i}=\text { Nov } 30}\left(X_{t_{i}}-E\left(X_{i}\right)\right)^{2}\right]^{1 / 2}
$$

$t_{i}=$ any day $t$ during $\mathrm{EP}_{\mathrm{i}}$ and $i=6 / 1$ through $11 / 30$ for each year, $\mathrm{y}=2004,2005, \ldots 2016 . \mathrm{X}_{t i}=$ the natural logarithmic function of changes in $\mathrm{X}_{\mathrm{i}}: \ln \left(\mathrm{X}_{\mathrm{i}, \mathrm{t}} / \mathrm{X}_{\mathrm{i}, \mathrm{t}-1}\right) \cdot \sigma\left(\mathrm{X}_{\mathrm{d}}\right)$ is the population standard deviation: $\left[1 / \mathrm{d} \sum_{\mathrm{t}=1}^{\mathrm{d}=\mathrm{n}_{\mathrm{y}} \text { or N }}\left(\mathrm{X}_{\mathrm{t}}-\mathrm{E}\left(\mathrm{X}_{\mathrm{d}}\right)\right)^{2}\right]^{1 / 2}$ for each $d=\mathrm{DP}_{\mathrm{Y}}$ and $\mathrm{DP}_{\mathrm{N}}$.

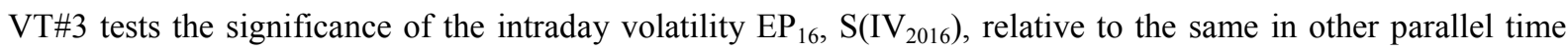
periods $\mathrm{S}\left(\mathrm{IV}_{\mathrm{i}}\right)$. VT\#4 tests the robustness of the results by evaluating each $\mathrm{S}\left(\mathrm{X}_{\mathrm{i}}\right)$ relative to the populations intraday volatility $\sigma\left(\mathrm{IV}_{\mathrm{d}}\right)$.

$$
\begin{gathered}
\mathrm{VT \# 3}=\mathrm{S}\left(\mathrm{IV}_{2016}\right) / \mathrm{S}\left(\mathrm{IV}_{\mathrm{i}}\right) \\
\mathrm{VT \# 4}=\mathrm{S}\left(\mathrm{IV}_{\mathrm{i}}\right) / \sigma\left(\mathrm{IV}_{\mathrm{d}}\right)
\end{gathered}
$$

Where: $\mathrm{S}\left(\mathrm{IV}_{\mathrm{i}}\right)$ is the sample volatility of intraday trading values during $\mathrm{EPi}$, or $\left[1 /(\mathrm{n}-1) \sum_{\mathrm{t}_{\mathrm{i}}=\operatorname{Jun} 1}^{\mathrm{n}_{\mathrm{i}}=\mathrm{Nov} 30}\left(\mathrm{IV}_{\mathrm{t}_{\mathrm{i}}}-\mathrm{E}\left(\mathrm{IV}_{\mathrm{i}}\right)\right)^{2}\right]^{1 / 2}$, and the inputs are defined as $\mathrm{IV}_{\mathrm{t}_{\mathrm{i}}}=\left(\mathrm{VX}_{\mathrm{H}, \mathrm{t}_{\mathrm{i}}}-\mathrm{VX}_{\mathrm{L}, \mathrm{t}_{\mathrm{i}}}\right) / \mathrm{VX}_{\mathrm{O}, \mathrm{t}_{\mathrm{i}}} \cdot \mathrm{IV}_{\mathrm{t}_{\mathrm{i}}}=$ the Intraday Volatility of $\mathrm{VX}$ on day $\mathrm{t}$ during $\mathrm{EPi}, \mathrm{VX}_{\mathrm{H}, \mathrm{t}_{\mathrm{i}}}=$ the Highest value of $\mathrm{VX}$ on day $\mathrm{t}$ during $\mathrm{EPi}, \mathrm{VX}_{\mathrm{L}, \mathrm{t}_{\mathrm{i}}}=$ the Lowest value of $\mathrm{VX}$ on day $\mathrm{t}$ during $\mathrm{EPi}, \mathrm{VX}_{\mathrm{O}, \mathrm{t}_{\mathrm{i}}}=$ the Opening value of $\mathrm{VX}$ on day $\mathrm{t}$ during EPi.

3.2 Predictive Tests (PT)

The predictive tests are based on the abnormal (AR) and cumulative abnormal returns (CAR) generated from equation 5:

$$
\ln \left(\mathrm{VX}_{\mathrm{d}, \mathrm{t}} / \mathrm{VX}_{\mathrm{d}, \mathrm{t}-1}\right)=\theta_{\mathrm{d}, \mathrm{o}}+\theta_{\mathrm{d}, 1} * \ln \left(\mathrm{VIX}_{\mathrm{d}, \mathrm{t}} / \mathrm{VIX}_{\mathrm{d}, \mathrm{t}-1}\right)+\mathrm{AR}_{\mathrm{d}, \mathrm{t}}
$$

where: $\ln \left(\mathrm{VX}_{\mathrm{d}, \mathrm{t}} / \mathrm{VX}_{\mathrm{d}, \mathrm{t}-1}\right)=$ the lognormal of the near VIX futures contract on day $t$, for population $d=\mathrm{DP}_{\mathrm{Y}}$ and $\mathrm{DP}_{\mathrm{N}}, \ln \left(\mathrm{VIX}_{\mathrm{d}, t} / \mathrm{VIX}_{\mathrm{d}, \mathrm{t}-1}\right)=$ the lognormal of the VIX spot index on day $t$ for population $d . \theta_{\mathrm{d}, \mathrm{o}}$ and $\theta_{\mathrm{d}, 1}=$ 
regression coefficients for population $d . \mathrm{AR}_{\mathrm{d}, \mathrm{t}}=$ the abnormal return prediction of $\ln \left(\mathrm{VX}_{\mathrm{d}, \mathrm{t}} / \mathrm{VX}_{\mathrm{d}, \mathrm{t}-1}\right)$ for parameters based on $d$, i.e., $\mathrm{AR}_{\mathrm{d}, \mathrm{t}}=\ln \left(\mathrm{VX}_{\mathrm{d}, \mathrm{t}} / \mathrm{VX}_{\mathrm{d}, \mathrm{t}-1}\right)-\left(\theta_{\mathrm{d}, \mathrm{o}}+\theta_{\mathrm{d}, 1} * \ln \left(\mathrm{VIX}_{\mathrm{d}, \mathrm{t}} / \mathrm{VIX}_{\mathrm{d}, \mathrm{t}-1}\right)\right.$.

$\mathrm{PT} \# 1$ and PT\#2 apply t-tests to determine if $\mathrm{AR}_{\mathrm{d}, \mathrm{t}_{\mathrm{i}}}$ and $\mathrm{CAR}_{\mathrm{d}, \mathrm{t}_{\mathrm{i}}}$ are insignificantly different from zero at each point in time based on population $d$ :

$$
\begin{aligned}
\text { Ho: } \mathrm{AR}_{\mathrm{d}, \mathrm{t}_{\mathrm{i}}} & =0 & & \text { Ha: } \mathrm{AR}_{\mathrm{d}, \mathrm{t}_{\mathrm{i}}} \neq 0 \\
\text { Ho: } \mathrm{CAR}_{\mathrm{d}, \mathrm{t}_{\mathrm{i}}} & =0 & & \text { Ha: } \mathrm{CAR}_{\mathrm{d}, \mathrm{t}_{\mathrm{i}}} \neq 0
\end{aligned}
$$

Each day during $\mathrm{EP}_{\mathrm{i}}, \mathrm{AR}_{\mathrm{d}, \mathrm{t}_{\mathrm{i}}}$ and $C A R_{d, t_{i}}$ are tested for significance relative to the volatility of the population, $\sigma\left(\mathrm{AR}_{\mathrm{d}}\right)$ and $\sigma\left(\mathrm{CAR}_{\mathrm{d}}\right)$ respectively.

$$
\begin{gathered}
\mathrm{PT \# 1}=\mathrm{AR}_{\mathrm{d}, \mathrm{t}_{\mathrm{i}}} / \sigma\left(\mathrm{AR}_{\mathrm{d}}\right) \\
\mathrm{PT} \# 2=\mathrm{CAR}_{\mathrm{d}, \mathrm{t}_{\mathrm{i}}} / \sigma\left(\mathrm{CAR}_{\mathrm{d}}\right)
\end{gathered}
$$

$\mathrm{PT \# 3}$ and PT\#4 apply F-tests to determine the significance of $\mathrm{S}\left(\mathrm{AR}_{\mathrm{d}, 16}\right)$ and $\mathrm{S}\left(\mathrm{CAR}_{\mathrm{d}, 16}\right)$ relative to each $\mathrm{S}\left(\mathrm{AR}_{\mathrm{d}, \mathrm{i}}\right)$ and $\mathrm{S}\left(\mathrm{CAR}_{\mathrm{d}, \mathrm{i}}\right)$ respectively, to test the hypotheses:

$$
\begin{aligned}
& \text { Ho: } \mathrm{S}\left(\mathrm{AR}_{\mathrm{d}, 16}\right) / \mathrm{S}\left(\mathrm{AR}_{\mathrm{d}, \mathrm{i}}\right)=1 \quad \text { Ha: } \mathrm{S}\left(\mathrm{AR}_{\mathrm{d}, 16}\right) / \mathrm{S}\left(\mathrm{AR}_{\mathrm{d}, \mathrm{i}}\right) \neq 1 \\
& \text { Ho: } \mathrm{S}\left(\mathrm{CAR}_{\mathrm{d}, 16}\right) / \mathrm{S}\left(\mathrm{CAR}_{\mathrm{d}, \mathrm{i}}\right)=1 \quad \mathrm{Ha}: \mathrm{S}\left(\mathrm{CAR}_{\mathrm{d}, 16}\right) / \mathrm{S}_{\left(\mathrm{CAR}_{\mathrm{d}, \mathrm{i}}\right) \neq 1} \\
& \mathrm{PT} \# 3=\mathrm{S}\left(\mathrm{AR}_{\mathrm{d}, 16}\right) / \mathrm{S}\left(\mathrm{AR}_{\mathrm{d}, \mathrm{i}}\right) \\
& \mathrm{PT} \# 4=\mathrm{S}\left(\mathrm{CAR}_{\mathrm{d}, 16}\right) / \mathrm{S}\left(\mathrm{CAR}_{\mathrm{d}, \mathrm{i}}\right)
\end{aligned}
$$

where: $\sigma\left(\mathrm{AR}_{\mathrm{d}}\right)=\left(\left(\frac{1}{\mathrm{~d}}\right) * \sum_{\mathrm{t}=1}^{\mathrm{d}=\mathrm{N} \text { or } \mathrm{n}_{\mathrm{y}}}\left(\left(\mathrm{AR}_{\mathrm{d}, \mathrm{t}}-\mathrm{E}\left(\mathrm{AR}_{\mathrm{d}}\right)\right)^{2}\right)^{1 / 2}, \sigma\left(\mathrm{CAR}_{\mathrm{d}}\right)=\left(\left(\frac{1}{\mathrm{~d}}\right) * \sum_{\mathrm{t}=1}^{\mathrm{d}=\mathrm{N} \text { or } \mathrm{n}_{\mathrm{y}}}\left(\left(\mathrm{AR}_{\mathrm{d}, \mathrm{t}}-\mathrm{E}\left(\mathrm{AR}_{\mathrm{d}}\right)\right)^{2}\right)^{1 / 2}\right.\right.$,

$\mathrm{S}\left(\mathrm{AR}_{\mathrm{d}, \mathrm{i}}\right)=\left(\left(\frac{1}{n_{i}}\right) * \sum_{\mathrm{t}_{\mathrm{i}}=\operatorname{Jun} 1}^{\mathrm{n}_{\mathrm{i}}=\operatorname{Nov} 30}\left(\left(\mathrm{AR}_{\mathrm{d}, \mathrm{t}_{\mathrm{i}}}-\mathrm{E}\left(\mathrm{AR}_{\mathrm{d}, \mathrm{i}}\right)\right)^{2}\right)^{1 / 2}, \mathrm{~S}\left(\mathrm{CAR}_{\mathrm{d}, \mathrm{i}}\right)=\left(\left(\frac{1}{\mathrm{n}_{\mathrm{i}}}\right) * \sum_{\mathrm{t}_{\mathrm{i}}=\operatorname{Jun} 1}^{\mathrm{n}_{\mathrm{i}}=\operatorname{Nov} 30}\left(\left(\mathrm{CAR}_{\mathrm{d}, \mathrm{t}_{\mathrm{i}}}-\mathrm{E}\left(\mathrm{CAR}_{\mathrm{d}, \mathrm{i}}\right)\right)^{2}\right)^{1 / 2}\right.\right.$.

\section{Results}

\subsection{Variation Test Results}

Table 4 displays the level values for $\mathrm{S}\left(\mathrm{VIX}_{\mathrm{i}}\right), \mathrm{S}\left(\mathrm{VX}_{\mathrm{i}}\right)$ and $\mathrm{S}\left(\mathrm{VVIX}_{\mathrm{i}}\right)$ with the corresponding F-test results for VT\#1 in columns $\mathrm{S}\left(\mathrm{VIX}_{16}\right) / \mathrm{S}\left(\mathrm{VIX}_{\mathrm{i}}\right), \mathrm{S}\left(\mathrm{VX}_{16}\right) / \mathrm{S}\left(\mathrm{VX}_{\mathrm{i}}\right)$ and $\mathrm{S}\left(\mathrm{VVIX}_{16}\right) / \mathrm{S}\left(\mathrm{VVIX}_{\mathrm{i}}\right)$. The findings show that $\mathrm{S}\left(\mathrm{VIX}_{16}\right)$ and $\mathrm{S}\left(\mathrm{VX}_{16}\right)$ are significantly higher than $\mathrm{S}\left(\mathrm{VIX}_{\mathrm{i}}\right)$ and $\mathrm{S}\left(\mathrm{VX}_{\mathrm{i}}\right)$ in seven of thirteen $\mathrm{EP}_{\mathrm{i}} \mathrm{s}$. Further, the level values of $\mathrm{S}\left(\mathrm{VIX}_{16}\right)$ and $\mathrm{S}\left(\mathrm{VX}_{16}\right)$ are exceeded by only one other year, 2011. The VVIX results indicating that the

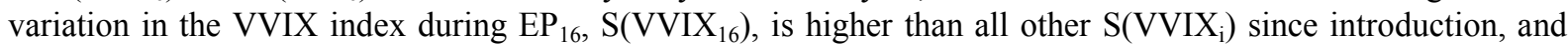
significantly so in seven of nine $\mathrm{EP}_{\mathrm{i}} \mathrm{S}$.

Table 4. Election Period Variation based on VT\#1

\begin{tabular}{lcccccc}
\hline $\mathrm{EP}_{\mathrm{i}}$ & $\mathrm{S}\left(\mathrm{VIX}_{\mathrm{i}}\right)$ & $\mathrm{S}\left(\mathrm{VIX}_{16}\right) / \mathrm{S}\left(\mathrm{VIX}_{\mathrm{i}}\right)$ & $\mathrm{S}\left(\mathrm{VX}_{\mathrm{i}}\right)$ & $\mathrm{S}\left(\mathrm{VX}_{16}\right) / \mathrm{S}\left(\mathrm{VX}_{\mathrm{i}}\right)$ & $\mathrm{S}\left(\mathrm{VVIX} \mathrm{i}_{\mathrm{i}}\right)$ & $\mathrm{S}\left(\mathrm{VVIX}_{16}\right) / \mathrm{S}\left(\mathrm{VVIX}_{\mathrm{i}}\right)$ \\
\hline 2004 & $4.56 \%$ & $\mathbf{2 . 2 3 1 9}$ & $4.61 \%$ & $\mathbf{1 . 5 4 1 2}$ & na & na \\
2005 & $5.01 \%$ & $\mathbf{2 . 0 3 0 0}$ & $3.30 \%$ & $\mathbf{2 . 1 5 2 6}$ & na & na \\
2006 & $6.03 \%$ & $\mathbf{1 . 6 8 9 0}$ & $4.65 \%$ & $\mathbf{1 . 5 2 6 7}$ & na & na \\
2007 & $9.02 \%$ & 1.1285 & $5.75 \%$ & 1.2341 & $5.31 \%$ & $\mathbf{1 . 4 2 0 7}$ \\
2008 & $9.51 \%$ & 1.0705 & $6.47 \%$ & 1.0980 & $5.20 \%$ & $\mathbf{1 . 4 4 9 2}$ \\
2009 & $5.34 \%$ & $\mathbf{1 . 9 0 6 9}$ & $4.05 \%$ & $\mathbf{1 . 7 5 1 1}$ & $3.98 \%$ & $\mathbf{1 . 8 9 5 5}$ \\
2010 & $6.05 \%$ & $\mathbf{1 . 6 8 1 6}$ & $5.20 \%$ & $\mathbf{1 . 3 6 5 0}$ & $4.21 \%$ & $\mathbf{1 . 7 9 0 4}$ \\
2011 & $\mathbf{1 0 . 4 8 \%}$ & 0.9714 & $\mathbf{7 . 4 2 \%}$ & 0.9571 & $6.20 \%$ & 1.2158 \\
2012 & $6.34 \%$ & $\mathbf{1 . 6 0 6 8}$ & $5.92 \%$ & 1.1983 & $4.14 \%$ & $\mathbf{1 . 8 2 1 6}$ \\
2013 & $6.03 \%$ & $\mathbf{1 . 6 8 8 5}$ & $4.14 \%$ & $\mathbf{1 . 7 1 6 3}$ & $5.15 \%$ & $\mathbf{1 . 4 6 3 3}$ \\
2014 & $7.76 \%$ & 1.3116 & $5.17 \%$ & $\mathbf{1 . 3 7 4 3}$ & $5.32 \%$ & $\mathbf{1 . 4 1 7 1}$ \\
2015 & $9.17 \%$ & 1.1105 & $6.39 \%$ & 1.1105 & $5.75 \%$ & 1.3121 \\
2016 & $\mathbf{1 0 . 1 8 \%}$ & 1.0000 & $\mathbf{7 . 1 0} \%$ & 1.0000 & $\mathbf{7 . 5 4 \%}$ & 1.0000 \\
\hline
\end{tabular}


Table 5 displays level values for $\sigma\left(\mathrm{VIX}_{\mathrm{Y}}\right), \sigma\left(\mathrm{VX}_{\mathrm{Y}}\right)$ and $\sigma\left(\mathrm{VVIX}_{\mathrm{Y}}\right)$ respectively. The results of the F-tests are revealed in columns $\mathrm{S}\left(\mathrm{VIX}_{\mathrm{i}}\right) / \sigma\left(\mathrm{VIX}_{\mathrm{N}}\right)-\mathrm{S}\left(\mathrm{VVIX}_{\mathrm{i}}\right) / \sigma\left(\mathrm{VVIX}_{\mathrm{Y}}\right)$ using volatility results from both DPY and DPN. Columns $\mathrm{S}\left(\mathrm{VIX}_{\mathrm{i}}\right) / \sigma\left(\mathrm{VIX}_{\mathrm{N}}\right)$ and $\mathrm{S}\left(\mathrm{VX}_{\mathrm{i}}\right) / \sigma\left(\mathrm{VX}_{\mathrm{N}}\right)$ show that $\mathrm{S}\left(\mathrm{VIX}_{\mathrm{i}}\right)$ and $\mathrm{S}\left(\mathrm{VX}_{\mathrm{i}}\right)$ are significantly greater than $\sigma\left(\mathrm{VIX}_{\mathrm{N}}\right)$ and $\sigma\left(\mathrm{VX}_{\mathrm{N}}\right)$ only for the 2011 and 2016 election periods. When the sample variances are compared to $\sigma\left(\mathrm{VIX}_{\mathrm{Y}}\right)$ and $\sigma\left(\mathrm{VX}_{\mathrm{Y}}\right)$ in columns $\mathrm{S}\left(\mathrm{VIX}_{\mathrm{i}}\right) / \sigma\left(\mathrm{VIX}_{\mathrm{Y}}\right)$ and $\mathrm{S}\left(\mathrm{VX}_{\mathrm{i}}\right) / \sigma\left(\mathrm{VX}_{\mathrm{Y}}\right)$, respectively, the single significant variable relative to $\sigma\left(\mathrm{VIX}_{\mathrm{Y}}\right)$ is the volatility of VIX during $\mathrm{EP}_{16}, \mathrm{~S}\left(\mathrm{VIX}_{16}\right)$. The volatility of VVIX, columns $\mathrm{S}\left(\mathrm{VVIX}_{\mathrm{i}}\right) / \sigma\left(\mathrm{VVIX}_{\mathrm{N}}\right)$ and $\mathrm{S}\left(\mathrm{VVIX}_{\mathrm{i}}\right) / \sigma\left(\mathrm{VVIX}_{\mathrm{Y}}\right)$, identifies the 2016 election period as the only one significantly greater than both $\sigma\left(\right.$ VVIX $\left._{\mathrm{N}}\right)$ and $\sigma\left(\mathrm{VVIX}_{\mathrm{Y}}\right)$. Tables 4 and 5 show that $\mathrm{EP}_{16}$ is a period of unprecedented uncertainty as indicated by consistently significant variations across all comparisons. Surprisingly, the results for $\mathrm{EP}_{16}$ overshadow even those for 2008, a year of significant economic disruption and bank failures.

Table 5. Election Period Variation based on VT\#2

\begin{tabular}{lccccccccc}
\hline EP & $\sigma\left(\mathrm{VIX}_{\mathrm{Y}}\right)$ & $\sigma\left(\mathrm{VX}_{\mathrm{Y}}\right)$ & $\sigma\left(\mathrm{VVIX}_{\mathrm{Y}}\right)$ & $\begin{array}{c}\mathrm{S}\left(\mathrm{VIX}_{\mathrm{i}}\right) / \\
\sigma\left(\mathrm{VIX}_{\mathrm{N}}\right)\end{array}$ & $\begin{array}{c}\mathrm{S}\left(\mathrm{VX}_{\mathrm{i}}\right) / \\
\sigma\left(\mathrm{VX}_{\mathrm{N}}\right)\end{array}$ & $\begin{array}{c}\mathrm{S}\left(\mathrm{VIX}_{\mathrm{i}}\right) / \\
\sigma\left(\mathrm{VIX}_{\mathrm{Y}}\right)\end{array}$ & $\begin{array}{c}\mathrm{S}\left(\mathrm{VX} \mathrm{i}_{\mathrm{i}}\right) / \\
\sigma\left(\mathrm{VX}_{\mathrm{Y}}\right)\end{array}$ & $\begin{array}{c}\mathrm{S}\left(\mathrm{VVIX}_{\mathrm{i}}\right) / \\
\sigma\left(\mathrm{VVIX}_{\mathrm{N}}\right)\end{array}$ & $\begin{array}{c}\mathrm{S}\left(\mathrm{VVIX}_{\mathrm{i}}\right) / \\
\sigma\left(\mathrm{VVIX}_{\mathrm{Y}}\right)\end{array}$ \\
\hline 2004 & $4.59 \%$ & $3.81 \%$ & na & 0.64761 & 0.8959 & 0.9931 & 1.2107 & na & na \\
2005 & $5.24 \%$ & $3.03 \%$ & na & 0.71201 & 0.6414 & 0.9563 & 1.0882 & na & na \\
2006 & $5.92 \%$ & $4.16 \%$ & na & 0.8558 & 0.9045 & 1.0188 & 1.119 & na & na \\
2007 & $8.27 \%$ & $5.22 \%$ & $5.45 \%$ & 1.28083 & 1.1189 & 1.0907 & 1.103 & 1.0536 & 0.9742 \\
2008 & $7.82 \%$ & $5.50 \%$ & $4.57 \%$ & 1.35023 & 1.2575 & 1.2164 & 1.175 & 1.0329 & 1.1393 \\
2009 & $5.57 \%$ & $4.38 \%$ & $3.64 \%$ & 0.75799 & 0.7885 & 0.9579 & 0.9257 & 0.7897 & 1.0916 \\
2010 & $7.30 \%$ & $5.46 \%$ & $4.59 \%$ & 0.85956 & 1.0116 & 0.8296 & 0.953 & 0.8361 & 0.9185 \\
2011 & $8.32 \%$ & $5.93 \%$ & $5.27 \%$ & $\mathbf{1 . 4 8 8 0 3}$ & $\mathbf{1 . 4 4 2 6}$ & 1.2598 & 1.2509 & 1.2312 & 1.1759 \\
2012 & $5.94 \%$ & $5.75 \%$ & $4.10 \%$ & 0.89957 & 1.1523 & 1.0659 & 1.0297 & 0.8217 & 1.0097 \\
2013 & $6.93 \%$ & $5.06 \%$ & $5.38 \%$ & 0.85603 & 0.8045 & 0.8699 & 0.8177 & 1.0229 & 0.9573 \\
2014 & $7.26 \%$ & $5.06 \%$ & $5.63 \%$ & 1.102 & 1.0047 & 1.0692 & 1.0212 & 1.0563 & 0.9454 \\
2016 & $8.71 \%$ & $5.75 \%$ & $6.28 \%$ & 1.30155 & 1.2434 & 1.0529 & 1.1113 & 1.1408 & 0.9155 \\
$\mathbf{2 0 0 4 - 1 6}$ & $\mathbf{7 . 0 4 \%}$ & $\mathbf{5 . 1 4 \%}$ & $\mathbf{5 . 0 4 \%}$ & $\mathbf{n a}$ & $\mathbf{n a}$ & $\mathbf{n a}$ & $\mathbf{n a}$ & $\mathbf{n a}$ & $\mathbf{n a}$ \\
\hline
\end{tabular}

The final volatility measures used to evaluate the volatility during the 2016 election period are based on VIX futures intraday volatility $\left(\mathrm{IV}_{\mathrm{i}}\right)$. Table 6 shows the level values for $\mathrm{S}\left(\mathrm{IV}_{\mathrm{i}}\right)$ and $\mathrm{S}\left(\mathrm{IV}_{\mathrm{Y}}\right)$ respectively. The results for VT\#3 are displayed in column $\mathrm{S}\left(\mathrm{IV}_{16}\right) / \mathrm{S}\left(\mathrm{IV}_{\mathrm{i}}\right)$ and show that the intraday volatility during EP16 is significantly greater than all other election periods examined. VT\#4, columns $\mathrm{S}\left(\mathrm{IV}_{\mathrm{i}}\right) / \sigma\left(\mathrm{IV}_{\mathrm{N}}\right)$ and $\mathrm{S}\left(\mathrm{IV}_{\mathrm{i}}\right) / \sigma\left(\mathrm{IV}_{\mathrm{Y}}\right)$, confirms the unusually high intraday volatility by showing $\mathrm{S}\left(\mathrm{IV}_{16}\right)$ is the only election period with significantly greater volatility compared to both $\sigma\left(\mathrm{IV}_{\mathrm{N}}\right)$ and $\sigma\left(\mathrm{IV}_{\mathrm{Y}}\right)$.

Overall, the four variation tests provide a strong case for significant investor uncertainty during the 2016 election period relative to twelve other parallel time periods.

Table 6. Intraday Volatility of VXi

\begin{tabular}{lccccc}
\hline $\mathrm{EP}_{\mathrm{i}}$ & $\mathrm{S}\left(\mathrm{IV}_{\mathrm{i}}\right)$ & $\mathrm{S}\left(\mathrm{IV}_{\mathrm{Y}}\right)$ & $\mathrm{S}\left(\mathrm{IV}_{16}\right) / \mathrm{S}\left(\mathrm{IV}_{\mathrm{i}}\right)$ & $\mathrm{S}\left(\mathrm{IV}_{\mathrm{i}}\right) / \sigma\left(\mathrm{IV}_{\mathrm{N}}\right)$ & $\mathrm{S}\left(\mathrm{IV}_{\mathrm{i}}\right) / \sigma\left(\mathrm{IV}_{\mathrm{Y}}\right)$ \\
\hline 2004 & 0.014944 & 0.015969 & $\mathbf{5 . 3 7 2 9}$ & 0.3467 & 0.9358 \\
2005 & 0.014302 & 0.017193 & $\mathbf{5 . 6 1 4}$ & 0.3318 & 0.8319 \\
2006 & 0.029695 & 0.026590 & $\mathbf{2 . 7 0 3 9}$ & 0.6889 & 1.1168 \\
2007 & 0.041698 & 0.040416 & $\mathbf{1 . 9 2 5 6}$ & 0.9674 & 1.0317 \\
2008 & 0.041912 & 0.064621 & $\mathbf{1 . 9 1 5 7}$ & 0.9723 & 0.6486 \\
2009 & 0.018529 & 0.022432 & $\mathbf{4 . 3 3 3 3}$ & 0.4299 & 0.8260 \\
\hline
\end{tabular}


Table 6 (continued). Intraday Volatility of VXi

\begin{tabular}{lccccc}
\hline $\mathrm{EP}_{\mathrm{i}}$ & $\mathrm{S}\left(\mathrm{IV}_{\mathrm{i}}\right)$ & $\mathrm{S}\left(\mathrm{IV}_{\mathrm{Y}}\right)$ & $\mathrm{S}\left(\mathrm{IV}_{16}\right) / \mathrm{S}\left(\mathrm{IV}_{\mathrm{i}}\right)$ & $\mathrm{S}\left(\mathrm{IV}_{\mathrm{i}}\right) / \sigma\left(\mathrm{IV}_{\mathrm{N}}\right)$ & $\mathrm{S}\left(\mathrm{IV}_{\mathrm{i}}\right) / \sigma\left(\mathrm{IV}_{\mathrm{Y}}\right)$ \\
\hline 2010 & 0.020925 & 0.048890 & $\mathbf{3 . 8 3 7 1}$ & 0.4854 & 0.4280 \\
2011 & 0.037961 & 0.033788 & $\mathbf{2 . 1 1 5 1}$ & 0.8807 & 1.1235 \\
2012 & 0.025697 & 0.027607 & $\mathbf{3 . 1 2 4 6}$ & 0.5961 & 0.9308 \\
2013 & 0.025283 & 0.031683 & $\mathbf{3 . 1 7 5 7}$ & 0.5865 & 0.7980 \\
2014 & 0.042718 & 0.041438 & $\mathbf{1 . 8 7 9 6}$ & 0.9910 & 1.0309 \\
2015 & 0.047469 & 0.042814 & $\mathbf{1 . 6 9 1 5}$ & 1.1012 & 1.1087 \\
$\mathbf{2 0 1 6}$ & $\mathbf{0 . 0 8 0 2 9 2}$ & $\mathbf{0 . 0 6 2 5 5 2}$ & $\mathbf{1 . 0 0 0 0}$ & $\mathbf{1 . 8 6 2 7}$ & $\mathbf{1 . 2 8 3 6}$ \\
$\mathbf{2 0 0 4 - 1 6}$ & $\boldsymbol{\sigma (}\left(\mathbf{I V}_{\mathrm{N}}\right)=$ & $\mathbf{0 . 0 4 3 1 0 5}$ & $\mathbf{V T \# 3}$ & $\mathbf{V T \# 4}$ & $\mathbf{V T \# 4}$ \\
\hline
\end{tabular}

\subsection{Predictive Test Results}

The Predictive Tests (PTs) are based on the results from 14 regressions; 13 apply $\mathrm{DP}_{\mathrm{Y}}$ and 1 applies $\mathrm{DP}_{\mathrm{N}}$. The regressions establish predictive values for $\ln \left(\mathrm{VX}_{\mathrm{d}, \mathrm{t}} / \mathrm{VX}_{\mathrm{d}, \mathrm{t}-1}\right)$, given contemporaneous values for $\ln \left(\mathrm{VIX}_{\mathrm{d}, \mathrm{t}} / \mathrm{VIX}_{\mathrm{d}, \mathrm{t}-1}\right)$. The focus of each PT is the significance of the abnormal returns $\left(\mathrm{AR}_{\mathrm{d}, \mathrm{t}_{\mathrm{i}}}\right)$ and cumulative abnormal returns $\left(C_{d, t_{i}}\right)$ during each election period. The $\mathrm{CARs}_{\mathrm{d}, \mathrm{t}_{\mathrm{i}}}$ are cumulated from the first trading day in June to the last trading day in November for each $\mathrm{EP}_{\mathrm{i}}$, but the parameters are estimated using $\mathrm{DP}_{\mathrm{Y}}$ and $\mathrm{DP}_{\mathrm{N}}$. The regression statistics are displayed in Table 1, for the interested reader.

Table 7 displays the results for PT\#1 and PT\#2 as the number of significant $A R_{Y, t_{i}}$ and $C A R_{Y, t_{i}}$ days, $n_{Y_{i}}^{*}$, as a proportion of the total number of days in the election period, $n_{i}, \boldsymbol{n}_{\boldsymbol{Y}_{\boldsymbol{i}}}^{*} / \boldsymbol{n}_{\boldsymbol{i}}$, using parameters generated from approximately 252 observations per year. Columns $\left.A R_{Y, i i} / S_{(A R}\right)$ indicate $\mathrm{EP}_{16}$ has the largest number of significant days at the $90 \%$ confidence level and the second the largest at the $95 \%$ level. The number of significant $C A R_{Y, t_{i}}$, displayed in columns $\mathrm{CAR}_{\mathrm{Y}, \mathrm{i}} / \mathrm{S}(\mathrm{CAR} \mathrm{Y})$, reveal no interesting results for 2016 . This finding suggests that the 2016 election period has a relatively large number of shocks that did not persist over time.

Table 7. Proportion of significant $\mathrm{AR}_{\mathrm{Y}, \mathrm{i}}$ and $\mathrm{CAR} \mathrm{R}_{\mathrm{ti \textrm {i }}}$ Days

\begin{tabular}{lcccc}
\hline \multirow{2}{*}{$\mathrm{EPi}$} & \multicolumn{2}{c}{$\mathrm{AR}_{\mathrm{Y}, \mathrm{ti}} / \mathrm{S}\left(\mathrm{AR}_{\mathrm{Y}}\right)$} & \multicolumn{2}{c}{$\mathrm{CAR}_{\mathrm{Y}, \mathrm{i}} / \mathrm{S}\left(\mathrm{CAR}_{\mathrm{Y}}\right)$} \\
\cline { 2 - 5 } & $90 \%$ & $95 \%$ & $90 \%$ & $95 \%$ \\
\hline 2004 & $3.94 \%$ & $3.15 \%$ & $13.39 \%$ & $7.87 \%$ \\
2005 & $2.34 \%$ & $2.34 \%$ & $26.56 \%$ & $10.94 \%$ \\
2006 & $7.03 \%$ & $5.47 \%$ & $21.09 \%$ & $17.19 \%$ \\
2007 & $7.81 \%$ & $6.25 \%$ & $41.41 \%$ & $29.69 \%$ \\
2008 & $6.30 \%$ & $5.51 \%$ & $21.26 \%$ & $15.75 \%$ \\
2009 & $5.47 \%$ & $3.91 \%$ & $12.50 \%$ & $3.91 \%$ \\
2010 & $6.25 \%$ & $3.91 \%$ & $12.50 \%$ & $6.25 \%$ \\
$\mathbf{2 0 1 1}$ & $7.81 \%$ & $5.47 \%$ & $\mathbf{5 0 . 7 8 \%}$ & $\mathbf{3 5 . 1 6 \%}$ \\
2012 & $5.56 \%$ & $3.17 \%$ & $42.86 \%$ & $26.98 \%$ \\
2013 & $6.30 \%$ & $4.72 \%$ & $21.26 \%$ & $15.75 \%$ \\
$\mathbf{2 0 1 4}$ & $8.66 \%$ & $\mathbf{7 . 8 7 \%}$ & $40.94 \%$ & $30.71 \%$ \\
2015 & $7.03 \%$ & $3.13 \%$ & $11.72 \%$ & $9.38 \%$ \\
$\mathbf{2 0 1 6}$ & $\mathbf{9 . 3 8 \%}$ & $7.03 \%$ & $13.28 \%$ & $8.59 \%$ \\
& $\mathbf{P T} \# \mathbf{1}$ & $\mathbf{P T} \# \mathbf{2}$ & & \\
\hline
\end{tabular}

Description: Parameters Estimated for Population $(\mathrm{N}=3,213)$. Parameters Estimated for each year separately, $\mathrm{n}$ » 252. 
Table 8 displays the results from repeating the first two PTs using DPN, rather than DPY. The results indicate the election period of 2008 has the highest number of significant $\mathrm{AR}_{\mathrm{N}, \mathrm{ti}}$ days at the $90 \%$ significance level and 2016 has the highest at the $95 \%$ level. The significance of the number of $\mathrm{CAR}_{\mathrm{N}, \mathrm{ti}}$ days remains unremarkable in 2016 , providing further support for frequent shocks that do not persist.

Table 8. Proportion of significant $A R_{N, t i}$ and $C A R_{N, t i}$ Days

\begin{tabular}{lcccc}
\hline \multirow{2}{*}{$\mathrm{EPi}$} & \multicolumn{2}{c}{$\mathrm{AR}_{\mathrm{N}, \mathrm{ti}} /\left(\mathrm{AR}_{\mathrm{N}}\right)$} & \multicolumn{2}{c}{$\mathrm{CAR}_{\mathrm{N}, \mathrm{ti}} /\left(\mathrm{CAR}_{\mathrm{N}}\right)$} \\
\cline { 2 - 5 } & $90 \%$ & $95 \%$ & $90 \%$ & $95 \%$ \\
\hline 2004 & $4.76 \%$ & $3.97 \%$ & $25.40 \%$ & $15.87 \%$ \\
2005 & $4.69 \%$ & $3.91 \%$ & $23.44 \%$ & $14.84 \%$ \\
2006 & $7.03 \%$ & $5.47 \%$ & $17.19 \%$ & $13.28 \%$ \\
2007 & $7.81 \%$ & $4.69 \%$ & $49.22 \%$ & $42.97 \%$ \\
$\mathbf{2 0 0 8}$ & $\mathbf{1 1 . 1 1 \%}$ & $6.35 \%$ & $26.19 \%$ & $23.02 \%$ \\
2009 & $3.91 \%$ & $3.91 \%$ & $32.81 \%$ & $11.72 \%$ \\
2010 & $6.25 \%$ & $5.47 \%$ & $11.72 \%$ & $7.03 \%$ \\
2011 & $4.69 \%$ & $1.56 \%$ & $58.59 \%$ & $51.56 \%$ \\
$\mathbf{2 0 1 2}$ & $7.94 \%$ & $5.56 \%$ & $\mathbf{7 4 . 6 0 \%}$ & $\mathbf{6 5 . 8 7 \%}$ \\
2013 & $1.57 \%$ & $1.57 \%$ & $11.02 \%$ & $6.30 \%$ \\
2014 & $8.66 \%$ & $7.03 \%$ & $33.86 \%$ & $18.90 \%$ \\
2015 & $9.45 \%$ & $6.30 \%$ & $13.39 \%$ & $10.24 \%$ \\
$\mathbf{2 0 1 6}$ & $10.94 \%$ & $\mathbf{7 . 0 9 \%}$ & $18.75 \%$ & $12.50 \%$ \\
& \multicolumn{2}{c}{$\mathbf{P T} \% \mathbf{2}$} \\
\hline
\end{tabular}

Description: Parameters Estimated for Population $(\mathrm{N}=3,213)$.

Table 9 depicts the results of PT\#3 and PT\#4, constructed to show the volatility of abnormal and cumulative abnormal returns during $\mathrm{EP}_{16}$ relative to the variabilities of each other election period based on annual parameters. Columns $\mathrm{S}\left(\mathrm{AR}_{\mathrm{Y}, \mathrm{i}}\right)$ and $\mathrm{S}\left(\mathrm{AR}_{\mathrm{Y}, 16}\right) / \mathrm{S}\left(\mathrm{AR}_{\mathrm{Y}, \mathrm{i}}\right)$ reveal the $\mathrm{S}\left(\mathrm{AR}_{\mathrm{Y}, 16}\right)$ is exceeded only by $\mathrm{S}\left(\mathrm{AR}_{\mathrm{Y}, 08}\right)$, however, significance is established in only five time periods. Columns $S\left(C A R_{Y, i}\right)$ and $S\left(C A R_{Y, 16}\right) / S\left(C A R_{Y, i}\right)$, confirm the lack of a significant or notable cumulative effect for $\mathrm{EP}_{16}$ based on annual parameters. The tests are repeated using $\mathrm{DP}_{\mathrm{N}}$ parameters with similar results.

Table 9. Residual Risk based on DPY

\begin{tabular}{ccccc}
\hline Year & $\mathrm{S}\left(\mathrm{AR}_{\mathrm{Y}, \mathrm{i}}\right)$ & $\mathrm{S}\left(\mathrm{AR}_{\mathrm{Y}, 16}\right) / \mathrm{S}\left(\mathrm{AR}_{\mathrm{Y}, \mathrm{i}}\right)$ & $\mathrm{S}\left(\mathrm{CAR} \mathrm{Y}_{\mathrm{i}}\right)$ & $\mathrm{S}\left(\mathrm{CAR} \mathrm{Y}_{\mathrm{Y}, 16}\right) / \mathrm{S}\left(\mathrm{CAR}_{\mathrm{Y}, \mathrm{i}}\right)$ \\
\hline 2004 & $3.69 \%$ & 1.1003 & $6.44 \%$ & 0.8199 \\
2005 & $2.90 \%$ & $\mathbf{1 . 4}$ & $5.58 \%$ & 0.9462 \\
2006 & $3.51 \%$ & 1.1567 & $7.49 \%$ & 0.7049 \\
2007 & $2.82 \%$ & $\mathbf{1 . 4 3 9 7}$ & $7.41 \%$ & 0.7126 \\
2008 & $4.22 \%$ & 0.9621 & $13.54 \%$ & 0.39 \\
2009 & $2.78 \%$ & $\mathbf{1 . 4 6 0 4}$ & $3.64 \%$ & $\mathbf{1 . 4 5 0 5}$ \\
2010 & $3.18 \%$ & 1.2767 & $6.53 \%$ & 0.8086 \\
2011 & $2.64 \%$ & $\mathbf{1 . 5 3 7 9}$ & $8.91 \%$ & 0.5926 \\
2012 & $3.82 \%$ & 1.0628 & $4.23 \%$ & 1.2482 \\
2013 & $2.00 \%$ & $\mathbf{2 . 0 3}$ & $3.54 \%$ & $\mathbf{1 . 4 9 1 5}$ \\
2014 & $3.05 \%$ & 1.3311 & $4.36 \%$ & 1.211 \\
\hline
\end{tabular}


Table 9 (continued). Residual Risk based on DPY

\begin{tabular}{ccccc}
\hline Year & $\mathrm{S}\left(\mathrm{AR}_{\mathrm{Y}, \mathrm{i}}\right)$ & $\mathrm{S}\left(\mathrm{AR}_{\mathrm{Y}, 16}\right) / \mathrm{S}\left(\mathrm{AR}_{\mathrm{Y}, \mathrm{i}}\right)$ & $\mathrm{S}\left(\mathrm{CAR}_{\mathrm{Y}, \mathrm{i}}\right)$ & $\mathrm{S}\left(\mathrm{CAR}_{\mathrm{Y}, 16}\right) / \mathrm{S}\left(\mathrm{CAR}_{\mathrm{Y}, \mathrm{i}}\right)$ \\
\hline 2015 & $3.19 \%$ & 1.2727 & $7.09 \%$ & 0.7447 \\
2016 & $4.06 \%$ & 1 & $5.28 \%$ & 1 \\
& & $\mathbf{P T} \# \mathbf{3}$ & & PT\#4 \\
\hline
\end{tabular}

The PT results point to a 2016 election period as one pocked by frequent unusual days, more so than other parallel time periods studied, but the shocks are not long-lasting.

\section{Conclusions}

The 2016 Paradox: a strong economy, strong stock market, low inflation, low unemployment and low VIX levels combined with high levels of anxiety, uncertainty and fear of the future. Incorrect conclusions regarding the state of investor sentiment were made because the focus was on a single parameter of risk-VIX levels. Using volatility tests and predictive test, this study demonstrates that VIX levels alone are insufficient, and at times misleading, in determining investor sentiment. The volatility tests showEP ${ }_{16}$ as a period of unusually high variations in VIX compared to other parallel non-election time periods. Similarly, intraday trading variations for VIX futures are exceptionally high during the 2016 election period. The predictive tests signal $\mathrm{EP}_{16}$ as a period with frequent abnormal shocks, without persistent echo. Together, the tests show that investor sentiment is better described by the distribution of VIX than the level.

This study provides evidence for significant investor disruption during the 2016 election period. The findings imply that in periods of economic calm, the levels of volatility indexes alone are insufficient and misleading in determining investor sentiment. The byzantine behavior of VIX during the 2016US Presidential election period, provides a unique opportunity to study VIX when economic concerns are not a dominate reason for investor anxiety. Under this scenario, VIX levels can remain low even while investor anxiety is high leading to incorrect conclusions regarding systematic risk levels.

The volatility tests indicate $\mathrm{EP}_{16}$ as a period of unusually high variations in the volatility indexes compared to other parallel non-election time periods. Similarly, intraday trading values for VIX futures, provides additional evidence in the form of exceptionally high intraday and volume variations.

The predictive tests consider the deviations of actual from predicted values for VIX futures. The results signal $\mathrm{EP}_{16}$ as a period with a relatively large number of significantly high abnormal return days but a low number of significant cumulative abnormal return days, indicating frequent shocks in $\mathrm{EP}_{16}$ that do not produce long-lasting results.

\section{References}

Dhaene, Jan, et al. (2012). FIX: The Fear Index-Measuring Market Fear. Topics in Numerical Methods for Finance, 37-55. US: Springer. https://doi.org/10.1007/978-1-4614-3433-7_4

Drechsler, Itamar. (2013). Uncertainty, Time-Varying Fear, and Asset Prices. The Journal of Finance, 68(5), 1843-1889. https://doi.org/10.1111/jofi.12068

Mamaysky, Harry, \& Paul Glasserman. (2016). Does Unusual News Forecast Market Stress?

Smales, Lee A. (2014). News sentiment and the investor fear gauge. Finance Research Letters, 11(2), 122-130. https://doi.org/10.1016/j.frl.2013.07.003

Tetlock, Paul C. (2007). Giving content to investor sentiment: The role of media in the stock market. The Journal of Finance, 62(3), 1139-1168. https://doi.org/10.1111/j.1540-6261.2007.01232.x

Traub, Heydon D. et al. (2000). Fear and greed in global asset allocation. The Journal of Investing, 9(1), 27-31. https://doi.org/10.3905/joi.2000.319396

Whaley, Robert E. (2000). The investor fear gauge. The Journal of Portfolio Management, 26(3), 12-17. https://doi.org/10.3905/jpm.2000.319728

\section{Copyrights}

Copyright for this article is retained by the author(s), with first publication rights granted to the journal. 
This is an open-access article distributed under the terms and conditions of the Creative Commons Attribution license (http://creativecommons.org/licenses/by/4.0/). 